\title{
3-ARYLIDENE-2-OXINDOLES AS QUINONE REDUCTASE II INHIBITORS
}

\section{E. Bezsonova', M. Dubar', D. Melekhina ${ }^{2}$, K. Evdokimov', V. Klochkov³ , E. Sokolova ${ }^{3}$}

${ }^{1}$ Department of Chemistry, Lomonosov Moscow State University, 119234, Russia Moscow, Leninskie gory St., 1.

2Department of Medicine Lomonosov Moscow State University, 119234, Russia Moscow, Leninskie gory St., 1.

${ }^{3}$ Department of Pharmacology and Bioinformatics, Volgograd State Medical University, 400131, Russia, Volgograd, Pavshikh Bortsov Sq. 1.

DOI: 10.19163/MedChemRussia2021-2021-392

E-mail:zetsu45999@mail.ru

Quinone reductase II (QR2) is an enzyme which exibits antioxidant, neuroprotective and chronobiotic properties. Previously we reported that 3-substituted-2-oindoles posess an affinity towards this molecular target and act as potential anti-glaucoma agents [1,2]. A number of novel compounds with 3-arylidene-2-oxindole scaffold were synthesized [3].

$\mathrm{R}_{1}=\mathrm{H}, \mathrm{CH}_{3}$

$\mathrm{R}_{5}=\mathrm{H}, \mathrm{NO}_{2}, \mathrm{Br}, \mathrm{AcNH}, \mathrm{CH}_{3} \mathrm{OC}(\mathrm{O}) \mathrm{NH}$, (2-furoyl) NH, BzNH

Aryl = 2-pyridyl, 2-thienyl, 2-furyl, 3-pyridyl, 3-Oh- $\mathrm{Ph}, 4$-pyridyl, 4-OH-Ph, 4-OMe-Ph, 4-OEt-Ph,

4- $\mathrm{NO}_{2}-\mathrm{Ph}$, 4-Br-Ph, 4-F-Ph, 3,4,5-tri-OMe-Ph, 3,5-di-OMe-4-OH-Ph, 1-Me-1H-pyrazol-4-yl, 3,5-di-

Me-pyrazol-4-yl, 1-Ph-1H-3,5-di-Me-pyrazol-4-yl, 3-ethoxycarbonyl-pyrazol-4-yl<smiles>[R3]CC1C(=O)N([R8])c2ccc([R3])cc21</smiles>

Configuration of novel compounds was determined by ${ }^{1} \mathrm{H}$ NMR spectrometry. E-configuration was found to be predominant in 4-subtituted benzylidene and 2-pyridinylmethylene derivatives, while in case of several pyrazole derivatives the stereoselectivity towards Z-configuration was observed. Obtained substances were tested as ligands of QR2 the and displayed prominent activity.

Inhibitory activity of selected compounds

\begin{tabular}{|c|c|c|c|c|c|}
\hline № & R1 & R5 & Aryl & $\%$ NQO2 Inhibition $(10 \mu \mathrm{M})$ & $\mathrm{IC}_{50}$ (NQO2) \\
\hline 1 & $\mathrm{H}$ & (2-furoyl)NH & 3,4,5-tri-OMe-Ph & 90,98 & 0,660 \\
\hline 2 & $\mathrm{H}$ & $\mathrm{AcNH}$ & 3,4,5-tri-OMe-Ph & 92,78 & 0,368 \\
\hline 3 & $\mathrm{H}$ & (2-furoyl)NH & 4-OH-Ph & 96,4 & 0,37 \\
\hline 4 & $\mathrm{H}$ & $\mathrm{CH}_{3} \mathrm{OC}(\mathrm{O}) \mathrm{NH}$ & $4-\mathrm{OH}-\mathrm{Ph}$ & 93,63 & 0,44 \\
\hline 5 & $\mathrm{CH}_{3}$ & $\mathrm{H}$ & 4-OH-Ph & 98,87 & 0,62 \\
\hline 6 & $\mathrm{H}$ & $\mathrm{H}$ & & 37,67 & 0,68 \\
\hline
\end{tabular}

This work was supported by the Russian Foundation for Basic Research, Project № 20-03-00915a

\section{References}

[1] M. S. Volkova, K. C. Jensen, N. A. Lozinskaya, S.E. Sosonyuk, M.V. Proskurnina, A. D. Mesecar, N. S. Zefirov, Bioorganic Med. Chem. Lett, 2012, 22(24), 7578-7581

[2] E. V. Zaryanova, N. A. Lozinskaya, O.V. Beznos, M. S. Volkova, N. B. Chesnokova, N.S. Zefirov, Bioorganic and Medicinal Chemistry Letters, 2017, 27, 3787-3793.

[3] N. A. Lozinskaya, D. A. Babkov, E.V. Zaryanova, E. N.Bezsonova, M. Efremov, M.D. Tsymlyakov, L. VAnikina, O. Yu, V. Borisov, V.N. Perfilova, I.N. Tyurenkov, M. V Proskurnina, Bioorg. Med. Chem, 2019, 27, 1804-1817. 